\title{
Finite Element Modeling of the Cortical Bone Region Using Clinical CT Images"
}

\author{
Hiroki NAKATSUCHI $^{* *}$ Shigeru TADANO ${ }^{* *}$ Masahiro TODOH ${ }^{* *}$ \\ Yukio NAKATSUCHI ${ }^{* * *}$ Shinichiro MORI ${ }^{* * * *}$ and Masahiro ENDO ${ }^{* * * *}$ \\ **Division of Human Mechanical Systems and Design, \\ Graduate School of Engineering, Hokkaido University, \\ N13 W8, Kita-ku, Sapporo, 060-8628 Japan \\ E-mail: nakatuchi@mech-me.eng.hokudai.ac.jp \\ ***Orthopedic Surgery, National Nagano Hospital, \\ 1-27-21 Midorigaoka, Ueda, 386-8610, Japan \\ ****Medical Physics, National Institute of Radiological Sciences, \\ 4-9-1 Anagawa Inage-ku, Chiba 263-8555, Japan
}

\begin{abstract}
Since the cortical bone has higher elastic modulus compared to the cancellous bone, its geometry is very important for stress analysis of bone structure. During finite element modeling of bone structure, cortical bone is generally determined as the region having higher $\mathrm{CT}$ values with respect to specific threshold value. However, it is difficult to determine the thin cortical bone regions by considering a specific threshold value. This study proposes a method to select regions of cortical bone from clinical CT images by considering CT value distributions of cortical and cancellous bone. Applying the method to bovine proximal femur, the mean error in cortical thickness compared to the actual bone was found to be less than one pixel $(0.39 \times 0.39 \mathrm{~mm})$. Hence, the proposed method could accurately determine the cortical bone regions from clinical CT images. The method was also applied to develop a finite element model with the precise cortical bone structure.
\end{abstract}

Key words: Biomechanics, Finite Element Model, Clinical CT Image, Cortical Bone Region, CT Value Distribution

\section{Introduction}

Understanding stress and deformation of bone structure is important in development and evaluation of joint prosthesis and for effective diagnosis of osteoporosis. To achieve this purpose, accurate three-dimensional modeling of bone structure based on X-ray CT images and finite-element analysis is required. Micro-CT images are widely used to analyze micro bone structure because of its high resolution ${ }^{(1)-(3)}$. However, the application of micro-CT images is limited to small bone samples. To analyze the whole bone structure including both cortical and cancellous bone, a finite element modeling from clinical CT images is appropriate.

The gray pixel level in CT images (CT value) depends on the X-ray absorption coefficient of the substance. Generally, hard tissue like bone has high X-ray absorption coefficients, and furthermore the cortical bone has higher bone densities and CT values than those of cancellous bone. The bone structure is modeled from the differences in their CT values - generally being distinguished at a specific threshold value ${ }^{(4)-(6)}$. But it is difficult to differentiate the two bone regions in cases when the thin cortical bone region has almost same CT values as that of cancellous bone. If a specific threshold value is considered, the 
geometrical error becomes large in such cases. Cortical bone having higher elastic modulus compared to cancellous bone, its geometry affects the stress distributions in the bone structure. Therefore, during FE analysis it is important to model the cortical bone geometry with high accuracy to predict the effects with less error.

This study proposes a new method to select the cortical bone region by considering characteristic CT value distributions in the boundary of cortical and cancellous bone. The cortical thickness obtained by applying the proposed method was compared with that of the corresponding section of actual bovine femur sample. Effectiveness of proposed method was confirmed according to good consistency of calculated cortical thickness with the actual thickness. Furthermore, a finite-element model was developed using proposed method.

\section{Materials and methods}

\subsection{X-ray CT Scan of bovine femur}

A left bovine femur sample $495 \mathrm{~mm}$ long weighing $2860 \mathrm{~g}$ was used (Fig. 1). Muscles, tendons and other soft tissues were removed from the surface. Three-dimensional cone beam CT scanner "Athena" (National Institute of Radiological Sciences, Japan) ${ }^{(7),(8)}$, which can give high resolution CT images, was used to scan the femur sample. Tube voltage 120 $\mathrm{kV}$ and tube current $200 \mathrm{~mA}$ were applied. The sample was scanned up to $166 \mathrm{~mm}$ from the top of greater trochanter in the distal direction. Pixel size of $0.39 \times 0.39 \mathrm{~mm}^{2}$ and slice thickness of $0.39 \mathrm{~mm}$ was obtained. Total of $426 \mathrm{CT}$ images were obtained. A single CT image was composed of $512 \times 512$ pixels. Bone mass phantom, B-MAS (Kyotokagaku.Co.Ltd, Kyoto, Japan) was also scanned. To avoid any differences in the CT values because of surrounding conditions, CT values $\left(V_{C T}\right)$ were converted to $\mathrm{CaCO}_{3}$ densities $\left(V^{*}\right)$. The mean CT values in each bone mass phantom $(80.17,133.47$ and 177.03 $\left.\mathrm{mg} / \mathrm{cm}^{3}\right)$ were calculated. CT values $\left(V_{C T}\right)$ and $\mathrm{CaCO}_{3}$ densities $\left(V^{*}\right)$ were linearly related as given in Eq.(1).

$$
V^{*}=k V_{C T}+l
$$

Here, $k=0.2915$ and $l=-302.8$. The converted values $\left(V^{*}\right)$ were used for rest of the work.

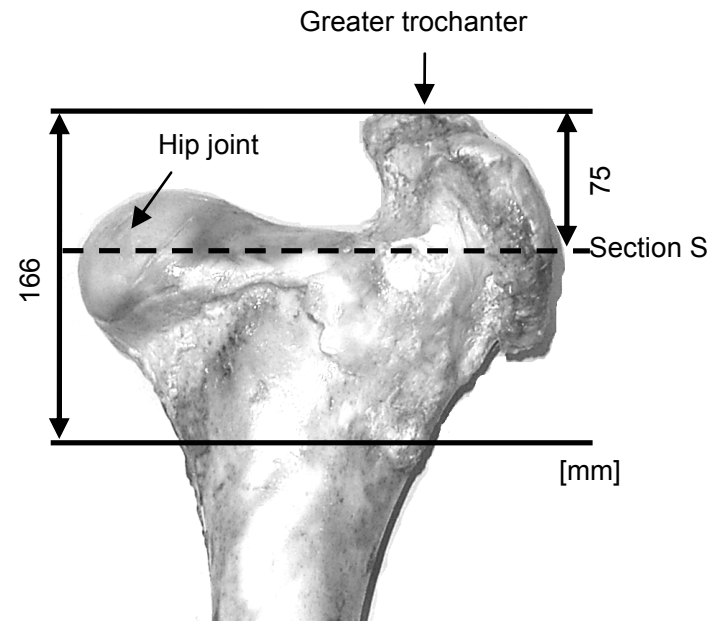

Fig. 1 Bovine proximal femur 


\subsection{Selection of bone region}

Since it is necessary to match the location of actual bone section with the CT image for comparison, the bovine femur was first sliced at a distance of $75 \mathrm{~mm}$ from the top of greater trochanter (Section S) as shown in Fig. 1 (see section 2.4). Among the CT images, each having $0.39 \mathrm{~mm}$ thickness, the $192^{\text {nd }} \mathrm{CT}$ image shown in Fig.2 was selected. It is obvious that the $\mathrm{CT}$ image consists of bone and non-bone regions and that cortical bone is observed in the outer part of the bone region. This section explains the procedure to separate bone and non-bone regions in the $\mathrm{CT}$ image.

From Fig. 2, it is clear that pixels in bone region have distinct higher CT values compared to non-bone region. Here, $3 \times 3$ pixels are considered a basic unit and bone or non-bone region is determined for each pixel in the unit. This process is indicated in Fig.3, where $C(i, j)$ shows the $\mathrm{CT}$ value on the center pixel $(i, j)$ of the unit. If all pixels in a unit are in the bone region, the mean $\mathrm{CT}$ value of 9 pixels is large and the standard deviation is small. When Eq.(2) is satisfied, all pixels in a unit are determined to be in the bone region. Then $m(i, j)$ is the mean CT value and $\sigma(i, j)$ is the standard deviation of the CT values.

$$
\frac{\sigma(i, j)}{m(i, j)} \leq \alpha
$$

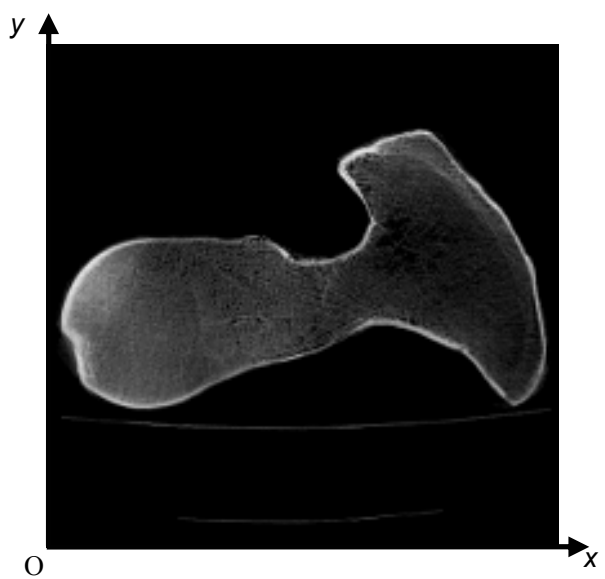

Fig. 2 CT image corresponding to section $\mathrm{S}$

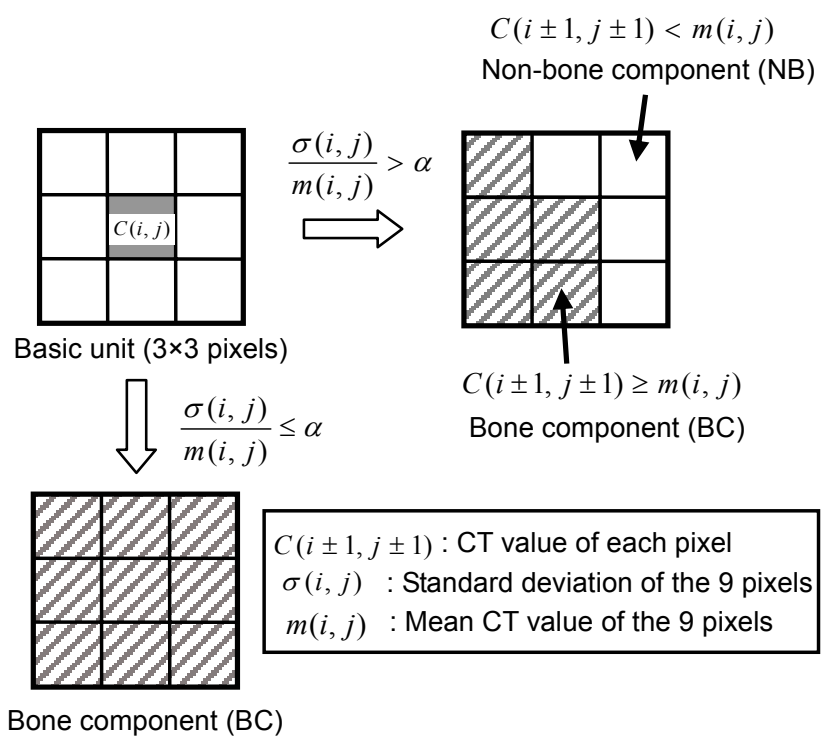

Fig. 3 Scheme for determining bone and non-bone components 
Here, $\alpha$ is a constant and is considered equal to 0.05 . Since $\sigma(i, j) / m(i, j)$ in the distinct cortical bone region of diaphysis has value less than 0.05 , we have set the same value for $\alpha$ here. If the condition in Eq.(2) is satisfied for each unit, all the pixels in the unit are considered as bone component (BC). If the condition given in Eq.(2) is not satisfied, it implies that pixels of both the bone and non-bone regions are included in the unit. Pixels greater than or equal to mean $\mathrm{CT}$ value are then considered as bone component (BC), whereas others as non-bone component (NB). Thus each pixel in the CT image is going to be examined nine times because of overlap. If the total number of calculation performed for a pixel results it as $\mathrm{BC}(N(i, j))$ for greater than or equal to five times, the pixel $(i, j)$ is considered to be a bone pixel. As shown in Table 1 , this condition $(N(i, j) \geqq 5)$ had low error of cortical thickness when compared to actual measurement value.

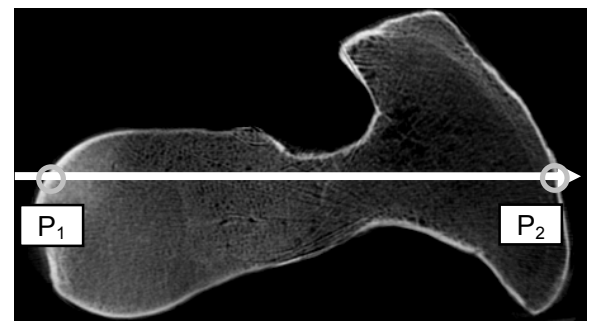

(a) CT image corresponding to section $\mathrm{S}$

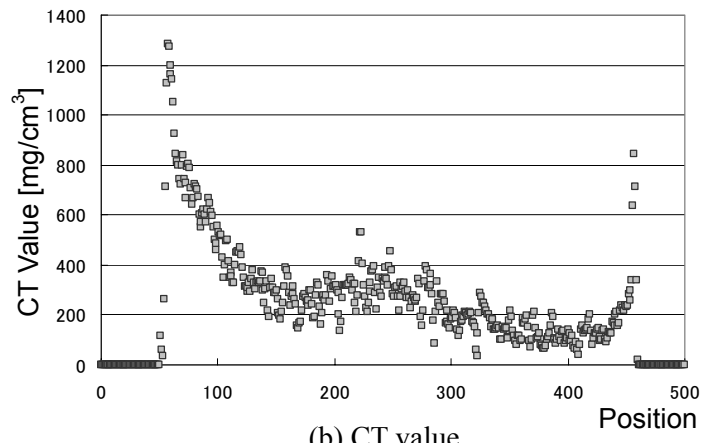

(b) CT value

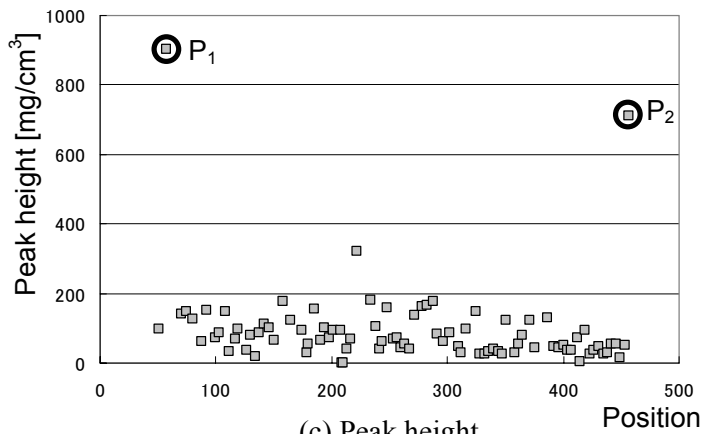

(c) Peak height

Fig. 4 Distribution of CT values and peak heights along $\mathrm{P}_{1} \mathrm{P}_{2}$

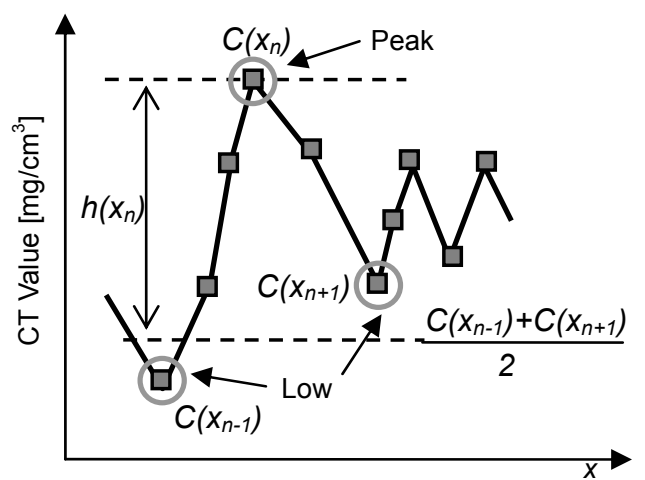

Fig. 5 Definition of peak height 


\subsection{Selection of cortical bone region}

The CT value distribution along the line $\mathrm{P}_{1} \mathrm{P}_{2}$ in Fig.4(a) is shown in Fig.4(b). The cortical bone region at both ends of Fig.4(b) has higher CT values than the cancellous bone region in the middle. However, it is difficult to indicate the boundary of cortical and cancellous bone region from the distribution of CT values in Fig.4(b) and it is difficult to separate the two bone regions using a specific threshold value. So, first the peak height is calculated from the $\mathrm{CT}$ value distribution. Peak points indicate the points having larger $\mathrm{CT}$ value compared to both adjacent points, whereas low points indicates the points that have less CT value compared to both adjacent points. Since the peak and low points are distributed alternately, a peak point $x_{n}$ exists between the low points $x_{n-1}$ and $x_{n+1}$ as shown in Fig. 5. The peak height is then defined from $C\left(x_{n}\right)$ and the mean CT value of the two lower points $\left(C\left(x_{n-1}\right), C\left(x_{n+1}\right)\right)$ at both sides of the peak point as given in Eq.(3).

$$
h\left(x_{n}\right)=C\left(x_{n}\right)-\frac{C\left(x_{n-1}\right)+C\left(x_{n+1}\right)}{2}
$$

Figure 4(c) shows the distribution of peak heights calculated from the CT values distribution in Fig.4(b). Because the peak heights of the cancellous bone region in Fig. 4(c) were less than $400 \mathrm{mg} / \mathrm{cm}^{3}$, the pixels satisfying $h\left(x_{n}\right)>400 \mathrm{mg} / \mathrm{cm}^{3}$ was defined as peak pixels in cortical bone.

Figure 6(a) and 6(b) show the distribution of only bone pixels and peak pixels respectively. Figure 6(c) is obtained by superimposing the two distributions. From this distribution, bone pixels which were continuously connected vertically and horizontally with a peak pixel were all assigned as cortical bone pixels. For instant, the region covered by arrows in Fig. 6(c) represents cortical bone pixels. The cortical bone region was determined in this way considering all the peak pixels. Figure 7 shows the cortical bone region obtained by this procedure. But still some errors in the form of noise exists, like those indicated by circles in Fig.7. Such noises are of one pixel width running along vertical and horizontal direction. This occurs because the pixels in cancellous bone and cortical bone regions are next to each other along vertical and horizontal direction.

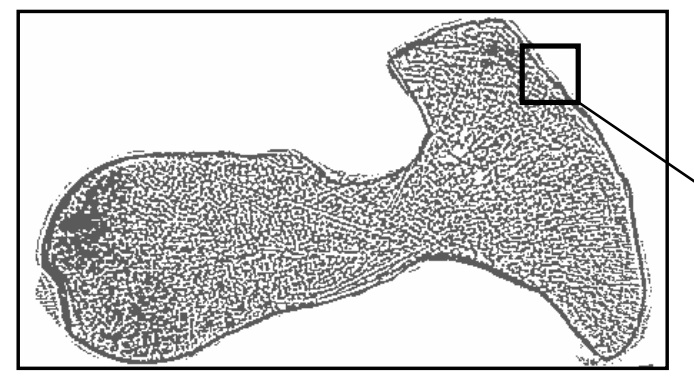

(a)
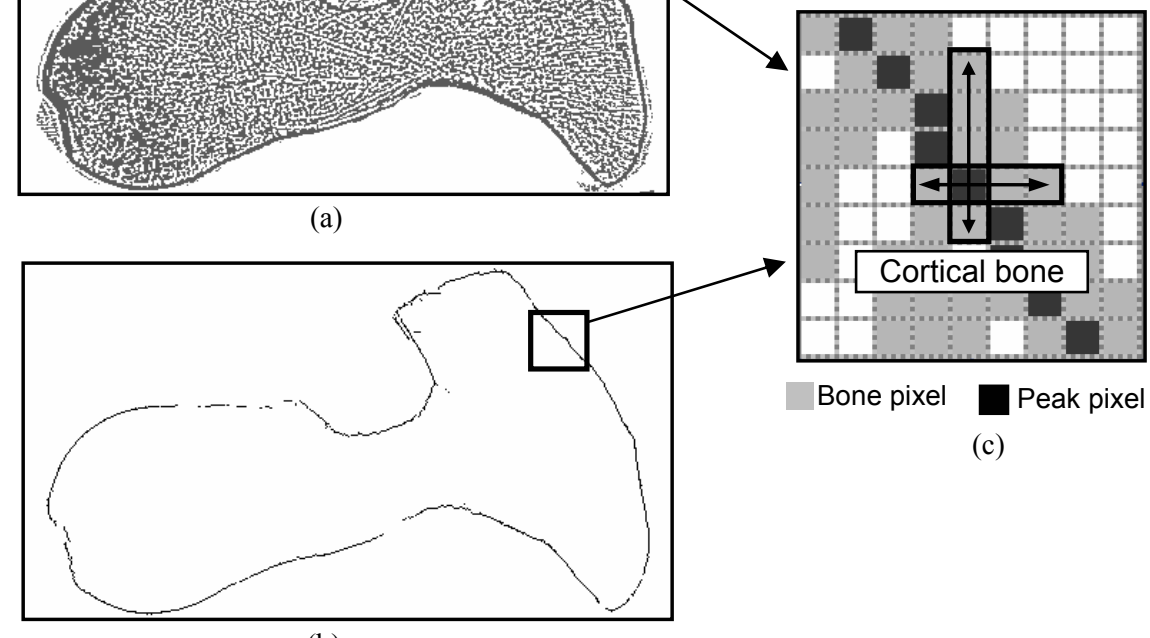

(c)

(b)

Fig. 6 Selection of cortical bone pixels. (a) Distribution of bone pixels, (b) Distribution of peak pixels, (c) Superimposition of the two distributions (a) \& (b) 
These noises are eliminated by the following. For $3 \times 3$ pixels with the tip of the noise at the center, only two pixels of cortical bone are included. Therefore, when the number of cortical bone pixels within $3 \times 3$ pixels is two, the central pixel is eliminated. The noise is eventually eliminated by iteration. Afterwards, the cortical bone region was smoothed by the dilation and erosion process. The boundary pixels of cortical bone region were enlarged by one pixel using the dilation process. Small holes in the region were filled and the roughness was smoothed. The boundary pixels were again reduced by one pixel using the erosion process. Such procedure of dilation and erosion has negligible effect in the thickness of cortical bone region.

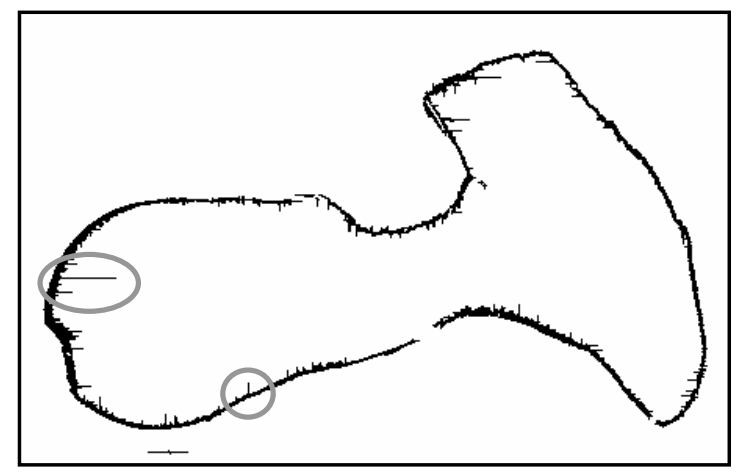

Fig. 7 Cortical bone region before smoothing process. Circles indicate noise examples.

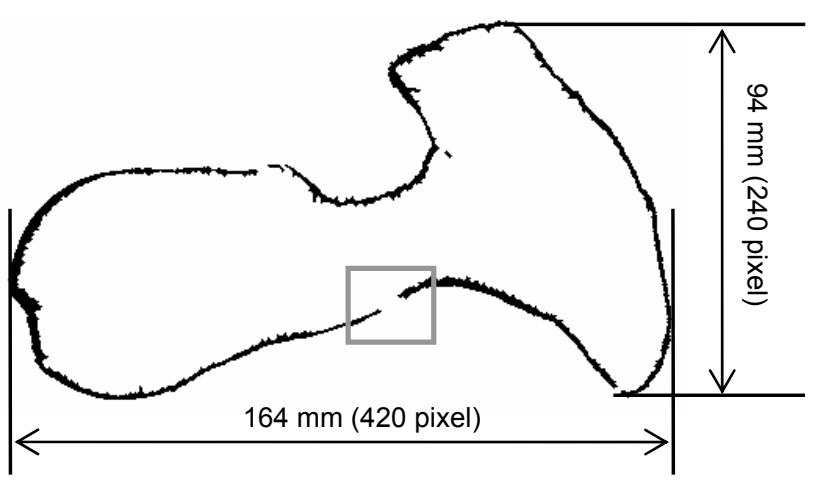

(a) Cortical bone region. Rectangle shows absence of cortical bone region.

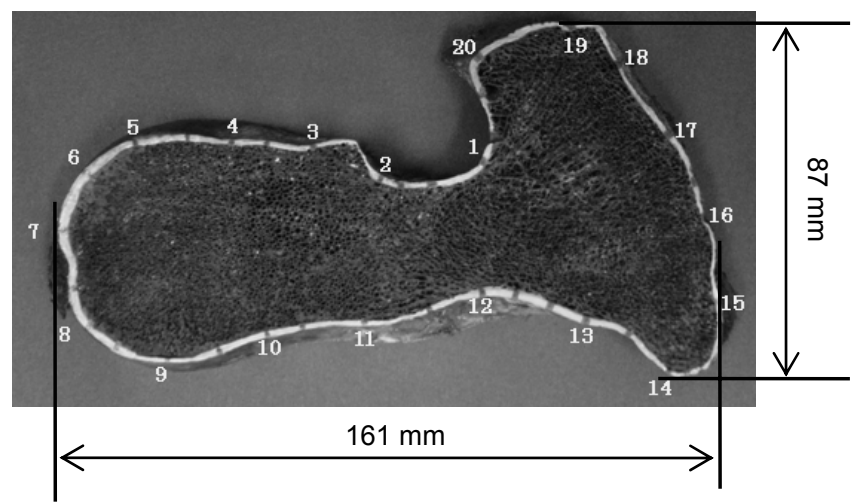

(b) Actual bone

Fig. 8 Cortical bone region corresponding to section $\mathrm{S}$ 


\subsection{Geometrical accuracy}

The accuracy of the cortical bone region described above was confirmed by comparing the cortical thickness to that of the actual bone section. A section of bovine femur perpendicular to bone axis at a distance $75 \mathrm{~mm}$ from the top of greater trochanter was sawed off. The location of this section is similar to the considered CT image (Fig.2). This was further sliced to have section equal to $15 \mathrm{~mm}$ thickness and bone marrow was removed using hot $\left(70-80^{\circ} \mathrm{C}\right)$ water. Figure $8(\mathrm{~b})$ shows the section obtained from the actual bone. Twenty positions were selected at equal intervals and the thickness of cortical bone at each position was measured as the distance from the outer surface of bone to the first bone-marrow cavity using a digital caliper. The thickness of the cortical bone region determined from the CT image as explained in previous section were also measured at the same 20 positions as in the actual bone. Since the pixels form a square region, the measurement directions were considered as horizontal, vertical or inclined at \pm 45 degrees wherever applicable according to the geometry of cortical region. For horizontal and vertical measurements, thicknesses were calculated by counting the number of cortical bone pixels and for inclined \pm 45 degrees, thicknesses were calculated by multiplying the number of cortical bone pixels by $\sqrt{2}$.

\subsection{FE modeling of cortical bone region}

Cortical bone regions of all the CT images were determined by this method. A three-dimensional geometric model of the cortical bone was made using the FE analysis software TRI/3D-BON (Ratoc System Engineering. Co. Ltd, Tokyo, Japan). The basic unit of the three-dimensional geometric model, voxel, is a cube (corresponding to pixel in the two-dimensional model), having size equal to $0.39 \times 0.39 \times 0.39 \mathrm{~mm}^{3}$. The FE model of cortical bone region was made by considering one voxel as one element.

Table 1 Comparison of the cortical bone thickness of the actual bovine proximal femur and this method

\begin{tabular}{|c|c|c|c|c|c|c|c|c|c|}
\hline \multirow{3}{*}{ Point } & \multicolumn{3}{|c|}{$\begin{array}{l}\text { Cortical bone } \\
\text { thickness }\end{array}$} & \multirow{3}{*}{$\begin{array}{c}\text { Difference } \\
\text { B-A } \\
\text { [pixel] }\end{array}$} & \multirow{3}{*}{ Point } & \multicolumn{3}{|c|}{$\begin{array}{l}\text { Cortical bone } \\
\text { thickness }\end{array}$} & \multirow{3}{*}{$\begin{array}{c}\text { Difference } \\
\text { B-A } \\
\text { [pixel] }\end{array}$} \\
\hline & \multicolumn{2}{|c|}{ Actual } & \multirow{2}{*}{$\begin{array}{c}\begin{array}{c}\text { This } \\
\text { method }\end{array} \\
\begin{array}{c}\mathrm{B} \\
\text { [pixel] }\end{array}\end{array}$} & & & \multicolumn{2}{|c|}{ Actual } & \multirow{2}{*}{$\begin{array}{c}\begin{array}{c}\text { This } \\
\text { method }\end{array} \\
\begin{array}{c}\mathrm{B} \\
\text { [pixel] }\end{array} \\
\end{array}$} & \\
\hline & {$[\mathrm{mm}]$} & $\underset{\text { [pixel] }}{A}$ & & & & [mm] & $\underset{[\text { pixel] }}{A}$ & & \\
\hline 1 & 1.52 & 3.90 & 5.00 & 1.10 & 11 & 0.80 & 2.05 & 3.00 & 0.95 \\
\hline 2 & 1.93 & 4.95 & 4.24 & -0.71 & 12 & 2.54 & 6.51 & 6.00 & -0.51 \\
\hline 3 & 0.85 & 2.18 & 3.00 & 0.82 & 13 & 2.06 & 5.28 & 5.00 & -0.28 \\
\hline 4 & 1.28 & 3.28 & 2.00 & -1.28 & 14 & 0.83 & 2.13 & 3.00 & 0.87 \\
\hline 5 & 1.57 & 4.03 & 5.66 & 1.63 & 15 & 1.48 & 3.79 & 3.00 & -0.79 \\
\hline 6 & 3.15 & 8.08 & 8.49 & 0.41 & 16 & 0.60 & 1.54 & 3.00 & 1.46 \\
\hline 7 & 3.04 & 7.79 & 6.00 & -1.79 & 17 & 1.48 & 3.79 & 4.24 & 0.45 \\
\hline 8 & 2.83 & 7.26 & 5.66 & -1.60 & 18 & 0.84 & 2.15 & 2.83 & 0.68 \\
\hline 9 & 1.47 & 3.77 & 3.00 & -0.77 & 19 & 1.31 & 3.36 & 3.00 & -0.36 \\
\hline 10 & 1.35 & 3.46 & 3.00 & -0.46 & 20 & 2.16 & 5.54 & 5.66 & 0.12 \\
\hline
\end{tabular}




\section{Results}

\subsection{Verification of geometrical accuracy}

Figure 8 (a) shows the cortical bone region determined by the proposed method. Figure 8(b) shows corresponding the actual bone section from the bovine femur. The white region in Fig.8(b) is the cortical bone region traced visually. The sizes of two sections, both the actual bone and the CT image, were approximately consistent as shown in Fig.8 (a) and (b). Cortical bone thicknesses obtained by this method and those of the actual bone at 20 different positions are given in Table 1. The maximum cortical thickness of actual bone was $3.15 \mathrm{~mm}$ (8.08 pixels) and the minimum value was $0.60 \mathrm{~mm}$ ( 1.54 pixels). The maximum error obtained was 1.79 pixels at position 7 . The mean square error was 0.98 pixels.

\subsection{FE model of cortical bone region}

Figure 9 shows the FE model of the cortical bone region developed by this method. Figures 9(a) and (b) show the posterior surface and coronal section of the FE model respectively. The cortical bone region is the white region in Fig.9(b), and the total number of elements was about 2 million.

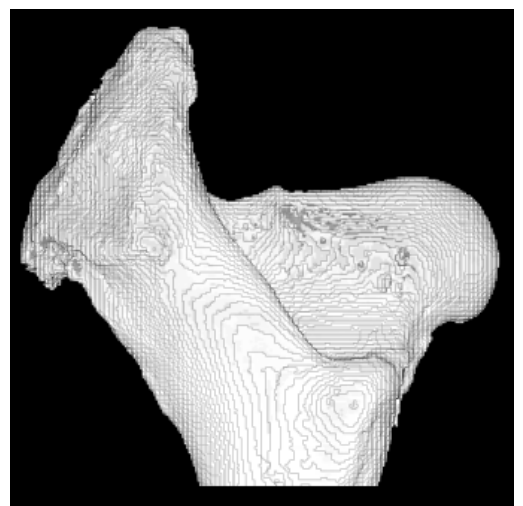

(a) Posterior surface

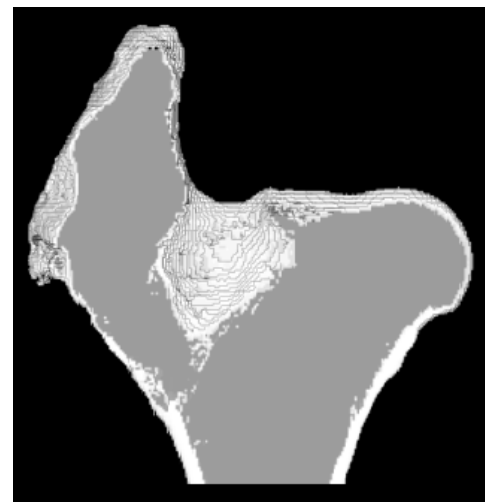

(b) Coronal section

Fig. 9 FE model of proximal bovine femur

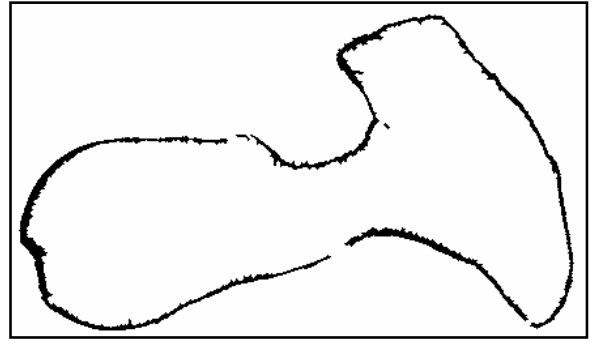

(a) This method

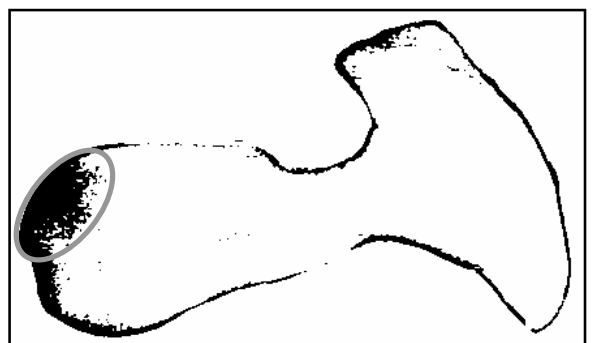

(b) Threshold value: 550

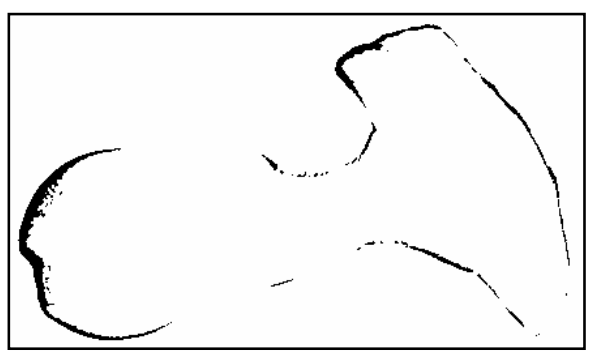

(c) Threshold value: 800

Fig. 10 Comparison of cortical bone regions determined by this method and by specific threshold values 


\section{Discussions}

\subsection{Comparison with models from specific threshold values}

Figure 10 shows comparison of the results of this method with those obtained considering specific threshold values. Figure 10(a) is the cortical bone region determined by the method developed in this work. Cortical region was extracted with threshold values from 500 to $1000 \mathrm{mg} / \mathrm{cm}^{3}$. Figure 10(b) and (c) represent the results with specific threshold values 550 and $800 \mathrm{mg} / \mathrm{cm}^{3}$ respectively. With the threshold value $550 \mathrm{mg} / \mathrm{cm}^{3}$, the cortical bone region at the femoral head was observed thicker than the actual bone. With the threshold value $800 \mathrm{mg} / \mathrm{cm}^{3}$, cortical bone regions were observed mostly lacking. Hence, the method developed in this work determined the geometry of cortical bone more effectively than the method based on specific threshold values.

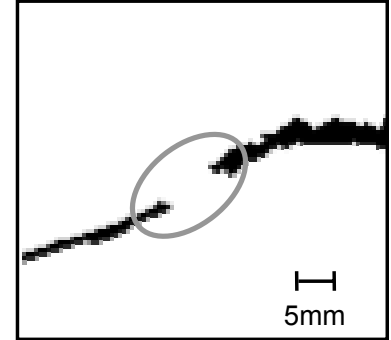

(a)

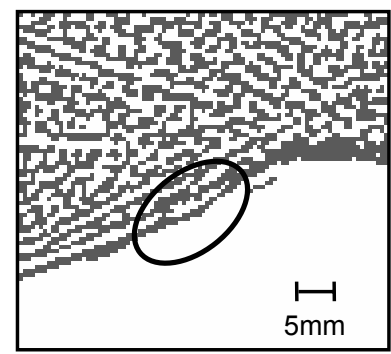

(c)

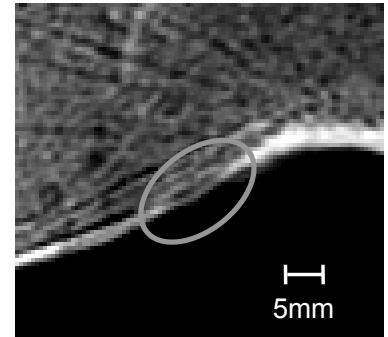

(b)

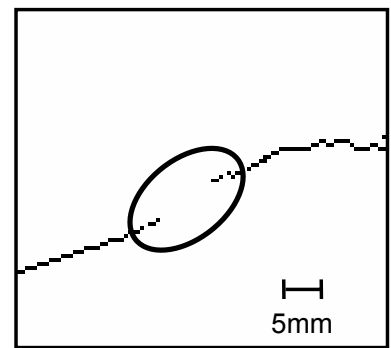

(d)

Fig. 11 Region with absent cortical bone (a) Cortical bone region image obtained by this method, (b) CT image, (c) Distribution of bone pixels, (d) Distribution of peak pixels

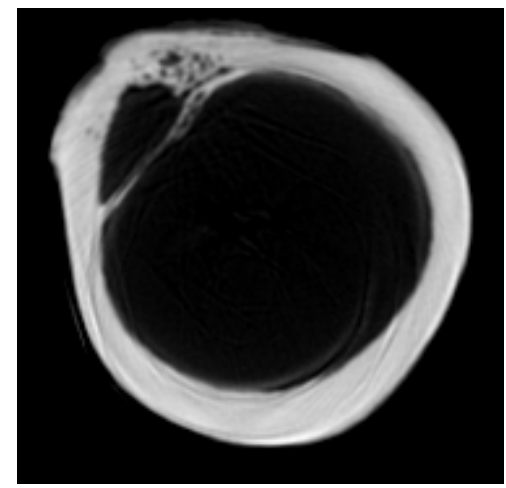

(a) CT image

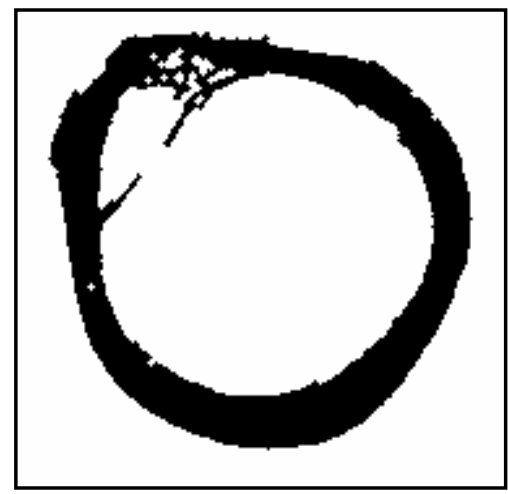

(b) Cortical bone region

Fig. $12 \mathrm{CT}$ image and cortical bone region in diaphysis 


\subsection{Useful range of the developed method}

The results obtained confirmed that the method introduced here could distinguish cortical bone from CT images with less than one pixel error compared to the actual bone. However a region where cortical bone was absent is seen in the results in Fig.8(a). Figure 11(a) shows the enlarged view around this region, and Fig.11(b) is the corresponding CT image. The CT image indicates presence of thin cortical bone in the region. The thickness of this thin cortical bone was measured to be $0.43 \mathrm{~mm}$ on the actual bone, which is nearly equal to one pixel of the CT image scanned in this study. Figures 11(c) and (d) show the distributions of bone and peak pixels around this region. Bone pixels are observed in this region (Fig.11(c)) but no peak pixels were found (Fig.11(d)). This indicates that the lack of cortical bone region results from a lack of peak pixels. This suggests the difficulty in selecting the pixels having values less than one pixel, when the CT values for both cortical and cancellous bone are close. Hence, this method is limited to the cortical bone region having thickness more than one pixel.

Figure 12 shows the CT image and corresponding cortical bone region in diaphysis of bone obtained using this method. Hence, this method also proves an accurate way to determine the cortical bone region which has higher pixel size.

\subsection{Application to human femur}

It has been reported that the average cortical thickness of human femoral neck is 1.46 $\mathrm{mm}$ in females (72 \pm 3$)$ and $1.77 \mathrm{~mm}$ in males $(72 \pm 3)^{(9)}$. The results in Table 1 show average cortical thickness equal to $1.65 \mathrm{~mm}$ for the bovine femur used in this study, which is almost equal to the cortical bone thickness of the human femoral neck. Therefore, it would be possible to apply this method to the human femur with similar precision.

The effectiveness of proposed method using the high-resolution of clinical CT was shown in this study. While, it is true that high X-ray exposure is needed to obtain high-resolution CT images in clinical situation. Therefore, it is also important to show the effectiveness of proposed method for low-resolution $\mathrm{CT}$ images by considering low X-ray exposure in future work.

\section{Conclusions}

This study proposes a method to distinguish the cortical bone region from clinical CT images precisely. From an application to the bovine proximal femur, we can conclude that:

1. The proposed method can distinguish the cortical bone region from clinical CT images effectively.

2. The mean difference in cortical thickness between this model and actual bone is less than one pixel $(0.39 \times 0.39 \mathrm{~mm})$.

\section{References}

(1) Steven K.B. et al, Smooth surface meshing for automated finite element model generation from 3D image data, Journal of Biomechanics, Vol.39, No7 (2006), pp. 1287-1295.

(2) Sandra J. S. et al, Prediction of fracture callus mechanical properties using micro-CT images and voxel-based finite element analysis, Bone, Vol.36, No.3 (2005), pp. 480-488

(3) Srinidhi N. Trabecular bone microdamage and microstructural stresses under uniaxial compression, Journal of Biomechanics, Vol.38, No4 (2005), pp. 707-716.

(4) Keyak, H.J. et al., Prediction of femoral fracture load using automated finite element modeling, Journal of Biomechanics, Vol.31, No2 (1998), pp.125-133.

(5) Schmitt, J. et al., Development of a hybrid finite element model for individual simulation of intertrochanteric osteotomies, Medical Engineering \& Physics, Vol.23, No.8 (2001), 
pp.529-539.

(6) Rubin, P.J. et al., Frictional interface micromotions and anisotropic stress distribution in a femoral total hip component, Journal of Biomechanics, Vol.26, No.6 (1993), pp.725-739.

(7) Endo, M. et al., Development and performance evaluation of the first model of 4-D CT-scanner, IEEE Transaction Nuclear Science, Vol.50, No.5 (2003), pp.1667-1671.

(8) Mori, S. et al., Physical performance evaluation of a 256-slice CT scanner for four-dimensional imaging, Medical Physics, Vol.31, No.6 (2004), pp.1348-1356.

(9) Kaptoge, S. et al., Effects of gender anthropometric variables, and aging on the evolution of hip strength in men and women aged over 65, Bone, Vol.32, No.5 (2003), pp.561-570 